\title{
First observation of a solar X-class flare in the submillimeter range with KOSMA
}

\author{
T. Lüthi ${ }^{1}$, A. Magun ${ }^{1}$, and M. Miller ${ }^{2}$ \\ ${ }^{1}$ Institute of Applied Physics, University of Bern, Sidlerstrasse 5, 3012 Bern, Switzerland \\ e-mail: thomas. luethi@mw.iap.unibe.ch, andreas .magun@mw. iap.unibe.ch \\ 2 I. Physikalisches Institut, Universität zu Köln, Zülpicher Strasse 77, 50937 Köln, Germany \\ e-mail:miller@ph1.uni-koeln.de
}

Received 24 April 2003 / Accepted 20 October 2003

\begin{abstract}
We present the first solar flare observations with the KOSMA submillimeter telescope at 230 and $345 \mathrm{GHz}$. The GOES X2.0 flare on April 12, 2001 was also observed at millimeter and centimeter wavelengths, as well as in soft and hard $\mathrm{X}$-rays. It exhibits both an impulsive phase of nonthermal gyrosynchrotron radiation and an extended phase of strong thermal free-free emission in the millimeter and submillimeter range. As in previous observations, a mismatch between the electron energy spectral indices, inferred from the millimeter and hard X-ray data, exists and is interpreted as a flattening of the energy spectrum above a break energy of several hundred keV. The observed thermal emission closely follows the shape of the $\mathrm{mm} / \mathrm{submm}$ flux density time profile predicted from soft X-ray observations. As the observed absolute flux densities exceed the predicted ones by a factor of $\sim 1.5-3.4$, both the $\mathrm{mm} / \mathrm{submm}$ emission and the soft X-rays must be thermal bremsstrahlung with a common energy source, but from locations with different plasma parameters. KOSMA observations allowed an estimate of source locations and sizes for the nonthermal and thermal sources. All of them coincide within 0.2 arcmin and with those seen in soft and hard X-rays. Surprisingly, the thermal submillimeter source diameters at 230 and $345 \mathrm{GHz}(42$ and 70 arcsec respectively) increase with frequency.
\end{abstract}

Key words. Sun: activity - Sun: flares - Sun: radio radiation - Sun: X-rays, gamma rays

\section{Introduction}

Millimeter and submillimeter observations of solar flares are important tools to diagnose the acceleration process of electrons at relativistic energies and the hot solar flare plasma. The main radiation mechanisms are gyrosynchrotron radiation and thermal bremsstrahlung (Kundu et al. 1990). The magnetic field in the solar corona usually does not exceed $2000 \mathrm{G}$ (White et al. 1991), corresponding to an electron gyrofrequency $v_{\mathrm{B}}$ of $\sim 6 \mathrm{GHz}$. A particle with Lorentz factor $\gamma$ typically emits at a harmonic number of $\gamma^{3}$, thus short millimeter waves are produced by $\gtrsim 0.5 \mathrm{MeV}$ electrons (White \& Kundu 1992; Kundu et al. 1994), the same electrons producing also the $\gamma$-ray continuum (Kawabata et al. 1982). Due to the limited sensitivity of $\gamma$-ray detectors, $\gamma$-ray observations have only been obtained for flares with large numbers of high energy electrons. Optically thin gyrosynchrotron emission in the $\mathrm{mm} /$ submm range, however, depends not only on the number of electrons, but also increases with the magnetic field strength. Therefore $\mathrm{mm} / \mathrm{submm}$ emission can often be observed even when the number of high energy electrons is too small to generate a detectable hard

Send offprint requests to: $\mathrm{T}$. Lüthi,

e-mail: thomas.luethi@mw.iap.unibe.ch
$\mathrm{X}$-ray/ $\gamma$-ray signature. Thus, radio observations at $\mathrm{mm} / \mathrm{submm}$ wavelengths are an attractive diagnostic tool for high energy electrons in solar flares (Trottet et al. 1998, 2000).

Nevertheless, the literature on solar flares at millimeter and submillimeter wavelengths is sparse. There are several reasons for the lack of observations: Besides the former inavailability of low-noise receivers at high frequencies, most flares exhibit a radio spectrum falling with increasing frequency. The thermal flux density of the quiet sun however is rising as $v^{2}$. This large background level is then modulated by the increasingly variable atmospheric attenuation, thus severely limiting the sensitivity of detection of solar millimeter and submillimeter flares (Nakajima et al. 1985; White \& Kundu 1992). Since 1984 regular patrol observations at $80 \mathrm{GHz}$ are available from a nulling interferometer at Nobeyama Solar Radio Observatory (Nakajima et al. 1985). A similar instrument at $89.4 \mathrm{GHz}$ is operated by the University of Bern since autumn 1999 (Lüthi 1999). These instruments cancel the quiet sun component, thus greatly improving the sensitivity for solar flare observations over the whole disk.

There are several reports of solar flare observations at short millimeter wavelengths during the 1969 solar maximum: Croom (1970) observed seven bursts at $71 \mathrm{GHz}$ in two and a 
half years of observations, including a very large event with a spectrum still rising at $71 \mathrm{GHz}$ (Croom \& Powell 1969). Shimabukuro $(1970,1972)$ reported several gradual flares at $90 \mathrm{GHz}$ with flat spectra at high frequencies and a good temporal correlation with soft X-ray observations. He explained the mm-emission as thermal bremsstrahlung. Other events seem to exhibit steeply rising spectra in the millimeter range, e.g. Akabane et al. (1973) and Kaufmann et al. (1985) at 73 and $90 \mathrm{GHz}$. Chertok et al. (1995) analysed several solar radio burst spectra in the range from 3 to $80 \mathrm{GHz}$. They focused on strong events at frequencies above $30 \mathrm{GHz}$ and found an extended group of events with increasing or flat spectra up to $80 \mathrm{GHz}$.

Kundu et al. (1990) reported the first spatially highly resolved interferometric observations of mostly weak solar flares at $86 \mathrm{GHz}$ with the Berkely-Illinois-Maryland Array (BIMA) in 1989. When compared to hard X-ray observations, many of the flares observed with BIMA indicate a flattening of the electron energy spectrum towards higher energies (e.g. Lim et al. 1992; Kundu et al. 1994; Silva et al. 1996; Raulin et al. 1999). A similar change of the spectral slope has been reported for combined observations of hard X-rays and microwaves (Marsh et al. 1981), whereas hard X-ray/ $\gamma$-ray spectroscopy in the energy range of $30 \mathrm{keV}-70 \mathrm{MeV}$ allowed the direct observation of broken energy spectra (e.g. Vestrand 1988; Rieger et al. 1998; Vilmer et al. 1999). Trottet et al. (1998, 2000) reported two events where the $\mathrm{cm} / \mathrm{mm}$ waves and the $\gamma$-rays above the break energy were produced by electron populations of the same spectral hardness. In 1999, a new solar dedicated millimeter and submillimeter telescope operating at 212 and $405 \mathrm{GHz}$ was installed in the Argentinean Andes (Kämpfer et al. 1997; Kaufmann et al. 1999). Trottet et al. (2002) reported a solar flare observed at $212 \mathrm{GHz}$, exhibiting both an impulsive phase of nonthermal gyrosynchrotron radiation and an extended phase of thermal emission. Unfortunately, these observations suffered from low sensitivity and lack of spectral information between 15 and $200 \mathrm{GHz}$.

Our solar observations at the Köln Observatory for Submillimeter and Millimeter Astronomy (KOSMA) began in late 2000. The $3 \mathrm{~m}$ telescope's dual-channel SIS-receiver is tunable from 210-270 and 330-365 GHz, with receiver noise temperatures of only 150 and $100 \mathrm{~K}$ respectively. Thus the instrument offers a significantly higher radiometric sensitivity than smaller telescopes with conventional uncooled Schottkyreceivers ( 2900 K at $212 \mathrm{GHz}$, see Kaufmann et al. 1999). KOSMA observations are complemented by our patrol observations, providing an unprecedented coverage of the radio spectrum between 8.4 and $345 \mathrm{GHz}$.

In this paper we present the first solar flare observations with KOSMA at 230 and $345 \mathrm{GHz}$ combined with the corresponding microwave and X-ray data. The instrumentation and calibration of the submillimeter data is described in Sect. 2. In Sect. 3 we analyse and discuss the nonthermal radio and hard X-ray emission during the impulsive phase of the event and the time-extended strong thermal emission. The last section presents our conclusions.

\section{Observations}

The flare under investigation occurred on April 12, 2001 at $\sim 10: 17$ UT (in the following all times are given in UT). The major event (GOES class X2.0) was located in the active region NOAA 9415 and was observed over a wide spectrum from hard X-rays down to radio wavelengths. The emission discussed in this paper covered the upper microwave spectrum with 8 frequencies from 8.4 to $345 \mathrm{GHz}$ as well as soft and hard X-rays observed with instruments aboard the GOES and Yohkoh spacecrafts (Fig. 1).

Microwave emission below $35 \mathrm{GHz}$ was first detected as a slow rise in intensity at $\sim 10: 14$ (phase I in Fig. 1), followed by significant emission with large spectral changes. Two phases with multiple injections become evident through two distinctive groups of peaks at $\sim 10: 17: 40$ and $\sim 10: 20: 25$ (phases II and III). After the impulsive phase an extended strong thermal emission (phase IV) reached its maximum at $\sim 10: 33$ and lasted at least until 12:45. From 10:58 to 11:40 flux density observations at 230 and $345 \mathrm{GHz}$ were interrupted in order to obtain solar maps (Fig. 2) of the thermal component, showing an observed maximum brightness temperature of $\sim 8300 \mathrm{~K}$ at both frequencies. Additional maps were taken at 9:15 well before the flare start. They serve as a quiet sun reference.

\section{Instrumentation}

Soft X-ray data was obtained by the dual-channel detectors (1.5-12.5 keV and 3-25 keV, corresponding to a wavelength of 1-8 $\AA$ and $0.5-4 \AA$ respectively) on the Geostationary Operational Environmental Satellites (GOES) with a time resolution of $3 \mathrm{~s}$. The flare was also seen with the soft X-Ray telescope (SXT) on the Yohkoh spacecraft, an imaging instrument in the $0.25-4.0 \mathrm{keV}$ range. Yohkoh flare mode observations cover the time interval from 10:13 to 10:39 and are continued on the next orbit after 11:47.

Hard X-ray emission was observed by the hard X-ray telescope (HXT) and the hard X-ray spectrometer (HXS) on Yohkoh. The former is an imager with four energy bands between 14 and $93 \mathrm{keV}$ and the latter a 32-channel spectrometer covering the energy range from 24 to $830 \mathrm{keV}$, operating with time resolutions of 2 and $4 \mathrm{~s}$ respectively.

The total flux at $\mathrm{cm}$ - and lower mm-wavelengths (8.4-50 GHz) was recorded with the patrol telescopes at Bumishus (Switzerland) with half-power beamwidths of $\sim 1$ degree and an integration time of 100 milliseconds. They (as well as all other radio telescopes used for this event) operate in total-power mode and use the quiet sun as a reference. Quiet sun flux densities from 8.4 to $19.6 \mathrm{GHz}$ were interpolated from RSTN measurements, and at higher frequencies calculated with the model of Croom (1979).

One of the difficulties with solar $\mathrm{mm} / \mathrm{submm}$ observations is the variability of atmospheric attenuation that increases strongly towards millimeter and submillimeter wavelengths. Therefore, and due to the large solar brightness temperature, the sensitivity for the detection of weak solar flares is severely limited. For the event under discussion this effect already becomes visible at 35 and $50 \mathrm{GHz}$ where observations suffered from bad weather (Fig. 1), resulting in non-solar flux 


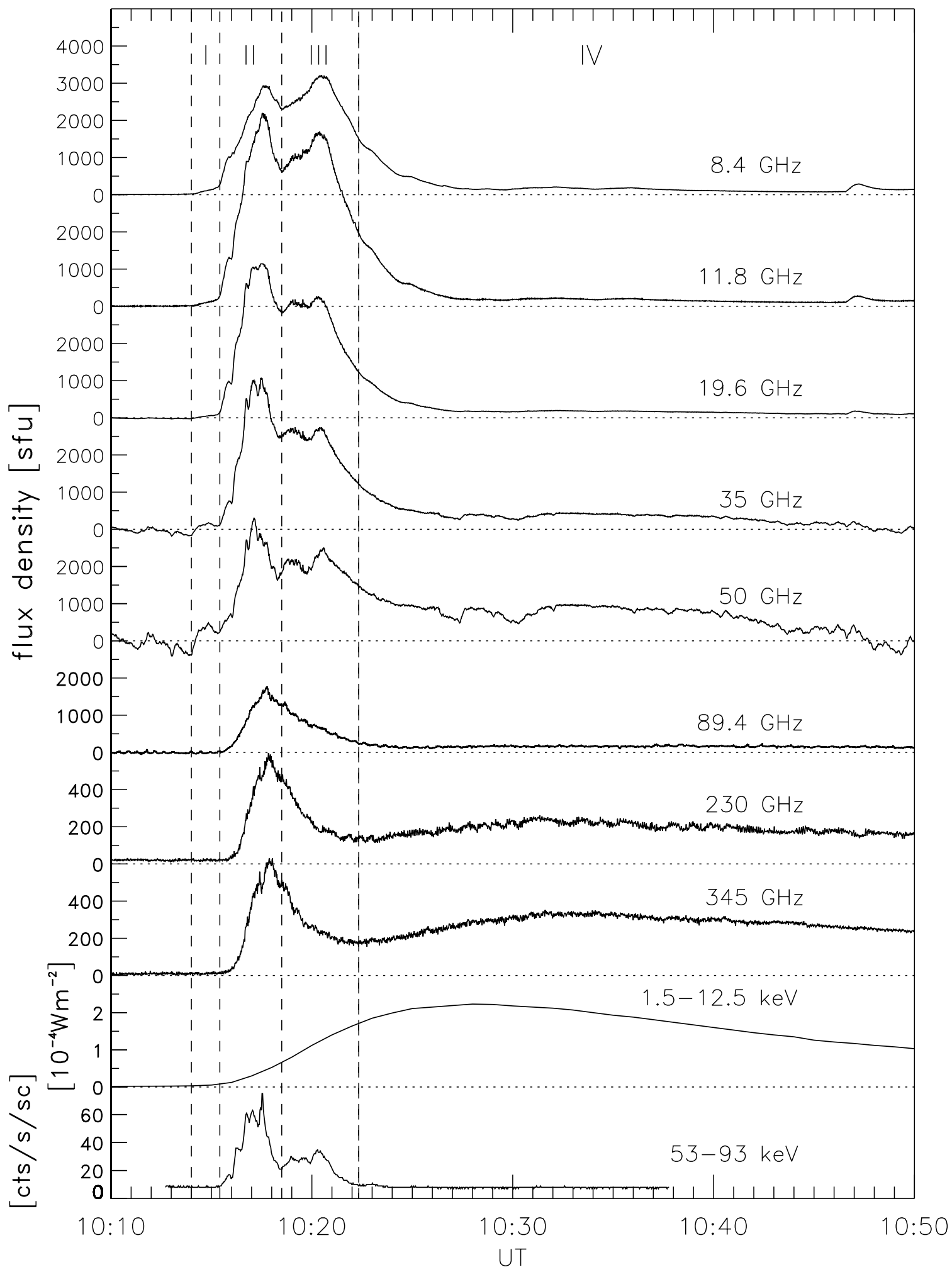

Fig. 1. Flux density time profiles at $8.4-50 \mathrm{GHz}$ (Bumishus observatory), $89.4 \mathrm{GHz}$ (nulling interferometer), $230 \mathrm{and} 345 \mathrm{GHz}$ (KOSMA), soft X-rays (GOES 10) and hard X-rays (Yohkoh HXT). The dashed vertical lines mark different episodes of the event. Yohkoh hard X-ray data ends with the beginning of satellite night at 10:39.

fluctuations of 5 and $7 \%$ (rms) respectively. Because of this effect, measurements at $89.4 \mathrm{GHz}$ are made by a nulling twoelement interferometer, set up to cancel the emission of the extended quiet sun. The flux of a solar flare, which in com- parison to the solar disk can be regarded as a point source, however is fully measured (Nakajima et al. 1985; Lüthi 1999). Our nulling-interferometer provides a half-power beamwidth of 53 arcmin and an integration time of 31 milliseconds. 

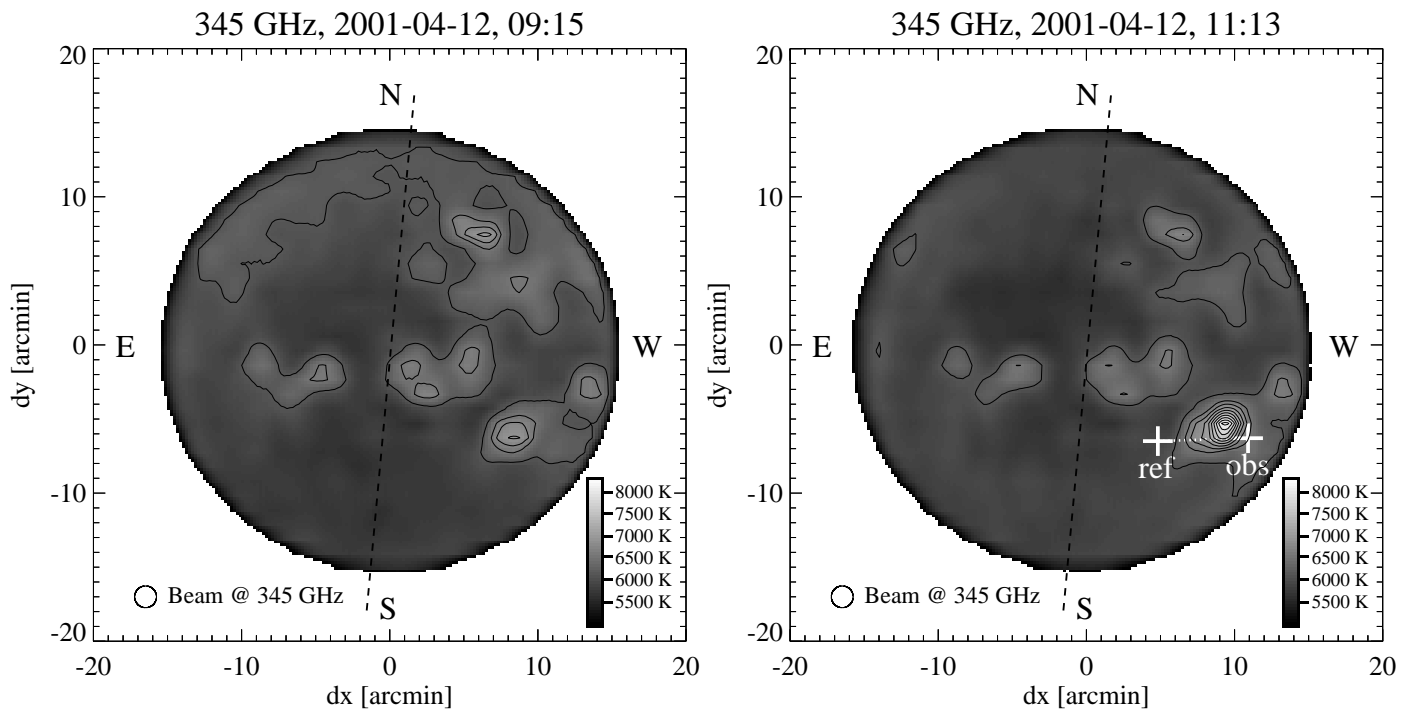

Fig. 2. Maps of the sun at $345 \mathrm{GHz}$ roughly 1 hour before and after flare start. At 11:13 thermal emission with a maximum measured brightness temperature of $\sim 8300 \mathrm{~K}$ was still visible. Contour levels begin at $6200 \mathrm{~K}$ with $300 \mathrm{~K}$ steps. Maps at $230 \mathrm{GHz}$ show the same features with lower resolution. The crosses on the right map are the end points of the subreflector scan from the observation ("obs") to the reference ("ref") position during maximum submm emission (10:17:54); the dashed lines show the rotation axis of the sun.

The resulting sensitivity is $\sim 35$ sfu under usual weather conditions, which is approximately one order of magnitude better than that of a single-dish radiometer with the same beamwidth.

The millimeter and submillimeter emission at 230 and $345 \mathrm{GHz}$ was observed with the KOSMA telescope on the Gornergrat in the Swiss Alps (Kramer et al. 1998). Low atmospheric attenuation at this high-altitude mountain site (3135 $\mathrm{m}$ a.s.l.) and the use of low-noise SIS-receivers make this instrument well suited for astronomical observations in the upper $\mathrm{mm} / \mathrm{submm}$-range. The SIS-mixers, as well as the large bandwidth $(1 \mathrm{GHz})$ of the receivers, result in a typical radiometric sensitivity of $\sim 2$ and $\sim 3$ solar flux units at 230 and $345 \mathrm{GHz}$ respectively, these values also including atmospheric effects. The integration time used was 2.3 milliseconds. The pointing accuracy of KOSMA is typically better than $\sim 10$ arcsec. Half-power beamwidths of the $3 \mathrm{~m}$-telescope are $117 \operatorname{arcsec}$ and $88 \operatorname{arcsec}$ at 230 and $345 \mathrm{GHz}$ respectively. Thus, the antenna beams cover a typical active region.

The active region NOAA 9415 was selected before tracking was started, as it showed the highest brightness temperature in our solar maps (Fig. 2, 09:15) and, from space weather predictions (Space Environment Center - online data), provided the greatest chance of flaring. A tiltable subreflector periodically deflected the antenna beams in cross-elevation by 6 arcmin from the target to a quiet reference region on the sun. The " $1 \mathrm{~s}$ on $/ 0.1 \mathrm{~s}$ off" observation cycle allowed the compensation of atmospheric attenuation changes with time scales above $1 \mathrm{~s}$. The absolute flux density calibration was obtained from hourly observations of the cold sky. Atmospheric conditions at Gornergrat were very stable (attenuation fluctuations $<1 \%$ ) with a zenith opacity of 0.18 and 0.48 at 230 and $345 \mathrm{GHz}$ respectively.

Unfortunately the flare occurred approximately 1.8 arcmin away from the initial observation region which became obvious only after the solar map at 11:13 had been analysed (Fig. 2).
Table 1. Source widths (half intensity) and flux density of the flare emission at $\sim 11: 10$ UT (extended phase). Note the significantly larger diameter at the higher frequency.

\begin{tabular}{lllll}
\hline \hline $\begin{array}{l}\text { Frequency } \\
{[\mathrm{GHz}]}\end{array}$ & $\begin{array}{l}\text { Beamwidth } \\
{[\operatorname{arcsec}]}\end{array}$ & $\begin{array}{l}\Theta_{\text {meas }} \\
{[\operatorname{arcsec}]}\end{array}$ & $\begin{array}{l}\Theta_{\text {source }} \\
{[\operatorname{arcsec}]}\end{array}$ & $\begin{array}{l}\text { Flux density } \\
{[\mathrm{sfu}]}\end{array}$ \\
\hline 230 & $117 \pm 7$ & 124 & $42 \pm 20$ & $112_{-4}^{+5}$ \\
345 & $88 \pm 5$ & 112 & $70 \pm 6$ & $196_{-9}^{+14}$ \\
\hline
\end{tabular}

A retargeting of the telescope on the flare after 11:40 and the data from the solar maps allowed us to estimate absolute flux values (see below).

\section{Flux calibration at 230 and $345 \mathrm{GHz}$}

Solar maps during the thermal phase ( $\sim 11: 13)$ were used to derive deconvolved source diameters and total flux values which are summarised in Table 1. Note that the source size increases with frequency. The source positions coincide within 0.2 arcmin and are in good agreement with those of the associated $\mathrm{H} \alpha$ and hard and soft X-ray emission (Fig. 4). The antenna beamwidths used for the deconvolution were determined from beam measurements on the solar limb.

In order to compensate atmospheric attenuation changes the antenna beams were moved periodically from the target ("obs" in Fig. 2) to a reference region ("ref"). However, when tracking the flare between 10:10 and 10:58 the antenna was pointed approximately one half-power beamwidth away from the emission region which reduced the observed flux. Thus, the measured flux density time profiles $S_{\text {det }}$ are too low and had to be corrected. Assuming symmetrical Gaussian profiles for the brightness temperature distribution and the antenna beam patterns, the flux density $S_{\text {Flare }}$ of the flare is given by

$S_{\text {Flare }}=S_{\text {det }} \frac{\rho_{\text {ant }}^{2}+\rho_{\text {source }}^{2}}{\rho_{\text {ant }}^{2}} \mathrm{e}^{r^{2} /\left(\rho_{\text {ant }}^{2}+\rho_{\text {source }}^{2}\right)}$, 

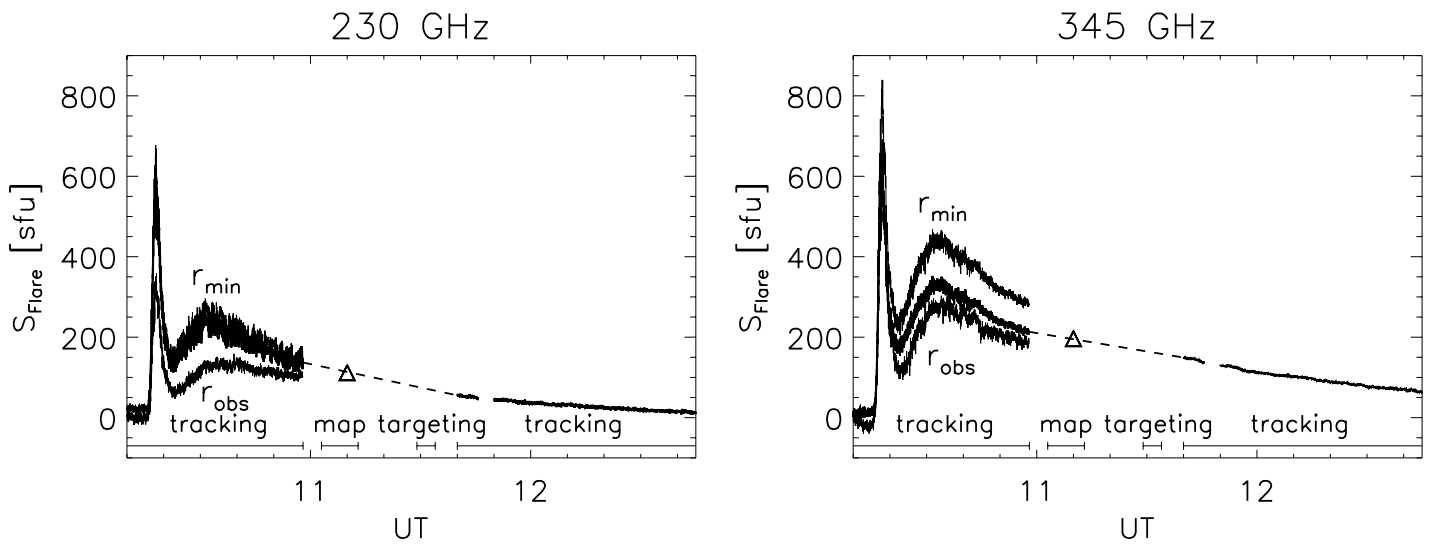

Fig. 3. Flux density time profiles from 10:10 to 10:58 for the observing position $\left(r_{\text {obs }}\right)$ and at minimum distance $\left(r_{\text {min }}\right)$ to the source. The time profile in between represents the corrected flux density obtained by scaling $S_{\text {det }}\left(r_{\min }\right)$ to fit the directly measured and deconvolved flux densities from the solar maps at 11:13 (triangles) and the re-targeted observation after 11:40.

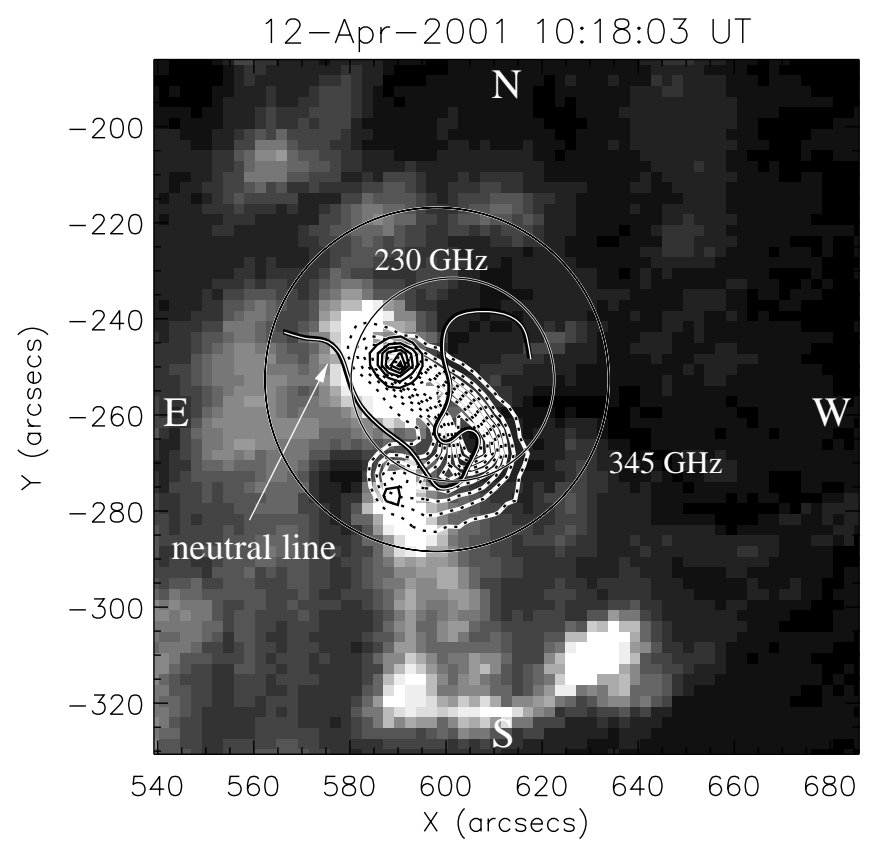

Fig. 4. Flare emission at 10:18:03 in $\mathrm{H} \alpha$ (background, provided by Kanzelhöhe observatory in Austria), Yohkoh SXT (dotted) and HXT 53-93 keV (solid contours). Also indicated are the half-power sources at 230 and $345 \mathrm{GHz}$ as seen in the solar maps at 11:13 (solarrotated to 10:18). The pointing accuracy is $\sim 25$ arcsec. There are several $\mathrm{H} \alpha$ kernels, two of which are connected by the soft X-ray loop across the magnetic neutral line (MDI/SOHO). The HPBW of the telescope is 117 and 88 arcsec at 230 and $345 \mathrm{GHz}$ respectively.

where $r$ are the angular separation of antenna beams and flare source, $\rho_{\text {ant }}$ and $\rho_{\text {source }}$ the $1 / e$-widths of the antenna pattern and the source respectively. Yohkoh SXT images showed no significant movement of the flaring loop throughout the event, thus a constant source position as seen in the solar maps at 11:13 was assumed. The angular distance $r$ between source and antenna beam centres changed during a subreflector scan from $r_{\text {obs }} \approx 1.8$ to a minimum of $r_{\text {min }} \approx 0.6$ arcmin, resulting in a peak in the observed flux density scan profiles. Thus, two measured time profiles of the flare can be extracted from the data: $S_{\text {det }}\left(r_{\text {obs }}\right)$ for the observation region "obs" and $S_{\text {det }}\left(r_{\min }\right)$ for the minimum distance $r_{\min }$. The constant position of the peak in the subreflector scan profiles also indicated a constant source position throughout the event. Its (deconvolved) halfpower width along the scan direction remained constant at $\leq 30$ and $70 \pm 15$ arcsec at 230 and $345 \mathrm{GHz}$ respectively. These values are consistent with the (thermal) source sizes seen in the solar maps. Therefore the latter were also used for the flux calibration during the impulsive phase. Corrections following Eq. (1) were then applied to both measured flux density time profiles. The corrected flux density $S_{\text {Flare }}\left(r_{\text {min }}\right)$ reached $2 S_{\text {Flare }}\left(r_{\text {obs }}\right)$ (Fig. 3 ) due to uncertainties of the source and antenna beam positions. The mismatch between the observations before 10:58 and the actual flux densities obtained from solar maps at $11: 13$ is also related to this. As the $\mathrm{mm} / \mathrm{submm}$ flux densities calculated from GOES soft X-ray observations indicate a smooth decline after $\sim 10: 35$ (see below), the flux densities at 11:10 and 11:40 were linearly extrapolated to 10:58. The flux density $S_{\text {det }}\left(r_{\text {min }}\right)$ from 10:10 to 10:58 was then scaled to match the extrapolated value at 10:58. The resulting time profiles at 230 and $345 \mathrm{GHz}$ are shown in Fig. 1.

It should be noted that Yohkoh images clearly show two hard X-ray sources at the footpoints of the soft X-ray loop (Fig. 4). Thus, the assumption of a single Gaussian radio source is only approximative. However, as the separation of the hard $\mathrm{X}$-ray sources of $\sim 25$ arcsec is small compared to the antenna half-power beamwidths, the corrected flux densities do not depend strongly on the actual source brightness distribution. The source diameter also turned out to be uncritical. Taking into account all uncertainties mentioned above, the resulting flux errors are estimated to ${ }_{-30}^{+20} \%$ and ${ }_{-40}^{+30} \%$ at 230 and $345 \mathrm{GHz}$ respectively.

\section{Analysis and interpretation}

The event under discussion can be divided into four phases (Fig. 1):

During phase I (10:14-10:15:25) emission was first detected at $10: 14$ as a slow rise in intensity at microwaves and hard $\mathrm{X}$-rays below $35 \mathrm{GHz}$ and $53 \mathrm{keV}$, respectively. The radio 
spectrum has a flat maximum, with the peak frequency rising from $\sim 10$ to $\sim 20 \mathrm{GHz}$.

Phase II (10:15:25-10:18:30) is characterised by a fast emission increase at microwaves, extending into the submillimeter range. It is accompanied by hard X-rays up to $\sim 200 \mathrm{keV}$. The centimeter and hard X-ray emission reached their maxima at $\sim 10: 17: 30$, while the $\mathrm{mm} / \mathrm{submm}$ emission peaked later at $\sim 10: 17: 54$. The radio spectrum is clearly non-thermal with a flat peak between 11.8 and $35 \mathrm{GHz}$ (Fig. 6).

Phase III (10:18:30-10:22:15) shows a second strong increase of emission below $89.4 \mathrm{GHz}$ and at hard X-rays. Its maximum occurred at $\sim 10: 20: 25$ and coincided with the maximum of the GOES time derivative. Above $50 \mathrm{GHz}$ we mainly observe the decay of electrons injected during phase II and only weak emission from the newly injected electrons.

Phase IV (10:22:15-12:45) is of thermal origin. After 10:22:15 the flux densities at 230 and $345 \mathrm{GHz}$ rose again and reached their maxima at $\sim 10: 33$. At this time the radio spectrum was nearly flat (Fig. 8). This emission lasted at least until 12:45 and became continuously harder. During the whole phase a strong soft X-ray emission evolved.

\subsection{X-ray emission}

\section{Soft X-rays}

The temporal evolution of the GOES $1.5-12.5 \mathrm{keV}$ count rate is represented in Fig. 1. Its maximum was reached at $\sim 10: 28: 30$ ( 10:25:00 for the 3-25 keV channel), well after the impulsive phase of the event. The time derivatives of both count rates peak at the time of maximum cm-emission in phase III. Assuming an isothermal source, the effective temperature $T_{\mathrm{e}}$ and emission measure $E M_{\mathrm{X}}$ of the hot flare plasma can be estimated from the two GOES energy bands using the procedure developed by Thomas et al. (1985). At 10:18:18, near the end of the first radio peak, $T_{\mathrm{e}}$ reached a maximum of $\sim 25 \times 10^{6} \mathrm{~K}$ with $E M_{\mathrm{X}}$ $\sim 2.8 \times 10^{49} \mathrm{~cm}^{-3}$. The latter reached its maximum of $\sim 2.0 \times$ $10^{50} \mathrm{~cm}^{-3}$ at 10:32:03 when $T_{\mathrm{e}}$ was $\sim 17 \times 10^{6} \mathrm{~K}$ and coincides with the maximum of the extended post-burst emission at 230 and $345 \mathrm{GHz}$.

The SXT-images show a single flaring loop across the magnetic neutral line seen in SOHO/MDI-images (Fig. 4). Its position and size remained almost constant throughout the observed time period (ending at satellite night). Depending on the SXT filter, the observed source area reached 180-260 $\operatorname{arcsec}^{2}$ at 10:33. At the beginning of the impulsive phase the main emission originated near the northern footpoint from an area of 40-60 $\operatorname{arcsec}^{2}$ and shifted towards the loop-top late in phase II. Unfortunately, no images, coincident in time with the solar maps at 230 and $345 \mathrm{GHz}$, are available. The first images after the satellite night show that the loop had approximately doubled in size $(\sim 11: 47)$.

\section{Hard X-rays}

The hard X-ray time profile at $53-93 \mathrm{keV}$ is in good agreement with the flux profiles observed at $19.6,35$ and $50 \mathrm{GHz}$

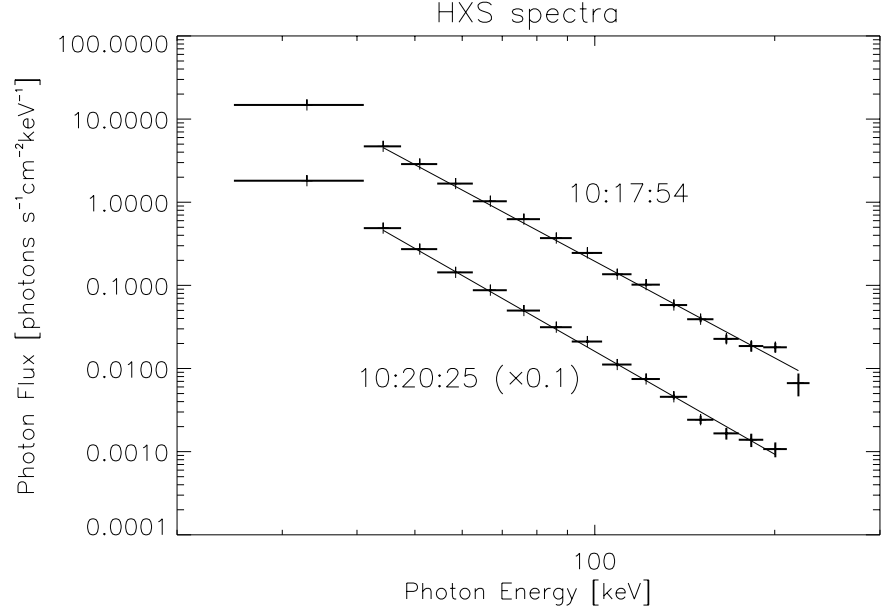

Fig. 5. HXS photon energy spectra at the times of peak 1 and $2(10 \mathrm{~s}$ average) with horizontal bars indicating the energy range of each HXS-channel and vertical bars the photon flux errors. Due to its poor energy resolution the lowest energy channel is not used for the fit. For clarity the spectrum at 10:20:25 has been multiplied with a factor of 0.1 . There are no significant counts above $\sim 200 \mathrm{keV}$.

(Fig. 1) as can be seen from the two distinctive groups of emission peaks during phases II and III and also from the common substructures. Using the sharp increase of emission at 10:16:00 as a marker, the delay between microwaves and hard X-rays is less than $2 \mathrm{~s}$ (time resolution of the HXT-instrument).

The 53-93 keV HXT-images show two sources which can be identified as footpoints of the soft X-ray loop (Fig. 4). They are also co-located with two $\mathrm{H} \alpha$ kernels in regions of opposite magnetic polarity. The northern hard X-ray source shows a 6 times stronger emission than the southern one which is hardly visible. This is consistent with the brightening of the northern part of the soft X-ray loop during phase II. There is no significant difference between the hard X-ray images during the first and second peak (phases II and III). The half-power source size during the impulsive phase of the event is $\sim 40 \operatorname{arcsec}^{2}$, corresponding to a source diameter of $\sim 7$ arcsec. After $\sim 10: 21$ the count rate becomes too low for image reconstruction. At lower photon energies additionally emission from the loop-top can be seen, until the 14-23 keV image nearly corresponds to the SXT-image.

The hard X-ray spectrum can be determined from the HXSdata, shown in Fig. 5. We assume that the energy spectrum of the hard X-ray photons follows a simple power-law of the form

$I(E)=I_{0}\left(\frac{E}{E_{0}}\right)^{-\gamma}$,

where $I$ is the count rate (photons $\mathrm{s}^{-1} \mathrm{~cm}^{-2} \mathrm{keV}^{-1}$ ), $E$ the photon energy, $I_{0}$ the count rate at the energy $E_{0}$ and $\gamma$ the photon spectral index. For $E_{0}=1 \mathrm{keV}$ the parameters $I_{0}$ and $\gamma$ were estimated using the HXS_FSP-program of the SSWIDL-package. Due to its poor energy resolution, the lowest energy channel is not used for the fit. At the time of maximum submm emission (10:17:54) we find $I_{0}=1.0 \times 10^{7}$ photons s${ }^{-1} \mathrm{~cm}^{-2} \mathrm{keV}^{-1}$ and $\gamma=3.9$ (Table 2). There is no indication for a break in the photon spectrum. 
Table 2. Power-law parameters of the fitted hard X-ray spectra during phases II and III. The spectral indices deduced from gyrosynchrotron modelling are also given.

\begin{tabular}{lll}
\hline \hline & $10: 17: 54$ & $10: 20: 25$ \\
\hline$I_{0}\left[\mathrm{~s}^{-1} \mathrm{~cm}^{-2} \mathrm{keV}^{-1}\right]$ & $1.0 \times 10^{7}$ & $2.7 \times 10^{7}$ \\
$\gamma$ & 3.9 & 4.1 \\
$\delta_{\mathrm{X}}$ & $4.9 \ldots 5.4$ & $5.1 \ldots . .6 .6$ \\
\hline$\delta_{\mathrm{r}}$ & $2.3 \pm 0.1$ & $3.5 \pm 0.2$ \\
\hline
\end{tabular}

In order to compare the hard X-ray and $\mathrm{mm} / \mathrm{submm}$ emission, we use a single loop model where electrons with a powerlaw energy spectrum are injected in the coronal part of a magnetic loop and precipitate towards its low-lying footpoints. The $\mathrm{mm} /$ submm radiation is gyrosynchrotron emission of relativistic electrons in the loop. Hard X-rays and $\gamma$-rays are produced by thin- or thick-target interactions, depending on the density of the medium and the time of the electrons spent in the loop (Brown 1971; Nitta et al. 1991). As the HXT-images clearly show footpoints, perfect trapping can be excluded. Thus, the spectral index of the electron population in the coronal loop is $\delta_{\mathrm{X}} \approx \gamma+1.5$ for free propagating electrons up to a few $100 \mathrm{keV}$ and $\delta_{\mathrm{X}} \approx \gamma+1$ at higher energies and for turbulently trapped electrons (e.g. Trottet et al. 1998, and references therein). The resulting electron energy spectral index at the time of maximum submm emission is therefore $\delta_{\mathrm{X}}=4.9-5.4$. During the second maximum of the microwave and hard X-ray emission (phase III) the HXS-spectrum is marginally softer with $I_{0}=2.7 \times 10^{7}$ photons s ${ }^{-1} \mathrm{~cm}^{-2} \mathrm{keV}^{-1}$ and $\gamma=4.1$, leading to $\delta_{\mathrm{X}}=5.1-5.6$.

\subsection{Radio emission}

Figure 1 shows that the microwave emission exhibits three distinctive components. An impulsive burst, visible up to submm wavelengths, was followed by a second peak of emission that was only observed at centimeter and longer millimeter wavelengths. Finally a long-lasting emission in the millimeter and submillimeter range was left.

While the microwave and hard X-ray emission during phase II starts and peaks simultaneously, the $\mathrm{mm} / \mathrm{submm}$ emission above $50 \mathrm{GHz}$ is clearly delayed (Fig. 7). To quantify this effect and to smooth out noise, running means over $10 \mathrm{~s}$ were directly compared. We find that the $89.4 \mathrm{GHz}$ onset and peak is delayed by $\sim 10 \mathrm{~s}$ with respect to the hard X-rays and microwaves, while emission at 230 and $345 \mathrm{GHz}$ is delayed by another $10 \mathrm{~s}$. Onset delays of the millimeter emission of several seconds relative to centimeter emission have been reported (Kundu et al. 1994; Lim et al. 1992; Trottet et al. 2002). They have been interpreted either as evidence that relativistic electrons producing millimeter and submillimeter emission are accelerated later than the microwave and hard X-ray-producing, lower energy electrons (Lim et al. 1992) or explained by a radio burst consisting of various successive injections of different spectral hardness of which only the hardest ones produce detectable high-frequency emission (Trottet et al. 2002).

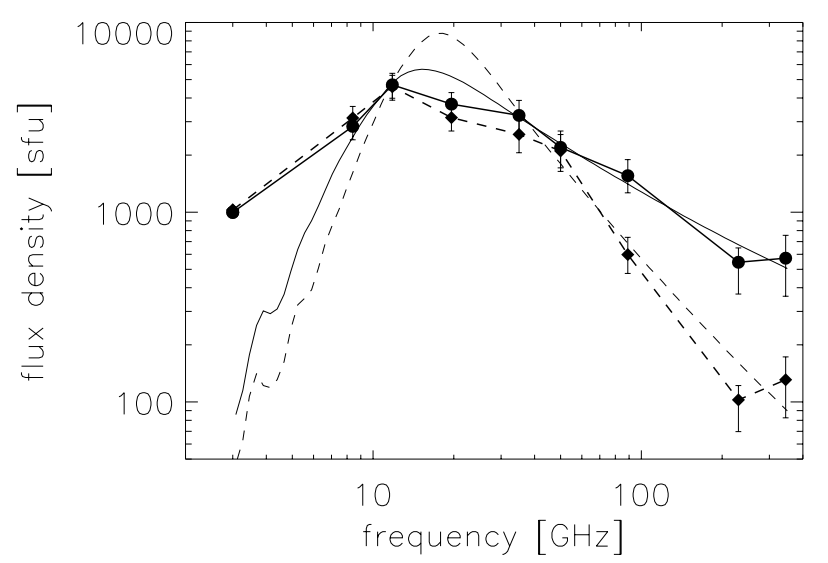

Fig. 6. Instantaneous radio spectra (10 s average) during the impulsive phase at 10:17:54 and 10:20:25 (dots and diamonds, respectively), including the $3.0 \mathrm{GHz}$ data point measured by the RT3 instrument at Ondrejov observatory (Czech Republic). The thermal emission component has been subtracted. The calculated model spectra for $B=500 \mathrm{G}$ that provide the best fit to the measured spectra above $35 \mathrm{GHz}$ are also shown (thin lines).

\section{Radio spectra during the impulsive phase}

GOES observations already indicate a substantial thermal emission component during the impulsive phase of the event (Fig. 9). They were used to estimate the thermal bremsstrahlung fluxes that amount to $\sim 5 \%$ of the $345 \mathrm{GHz}$ flux density at the maximum of the first and $\sim 40 \%$ at the maximum of the second group of peaks (using the scaled fluxes, see Fig. 8). These thermal components have been subtracted prior to further spectral analysis. The resulting radio spectrum during the impulsive phase of the flare clearly shows non-thermal signatures (Fig. 6). A nearly flat spectrum between 11.8 and $35 \mathrm{GHz}$ indicates the presence of source inhomogeneities (Klein \& Trottet 1984). This interpretation is further supported by the spectral index below the spectral maximum ( 1) which can only be explained by source inhomogeneities (Dulk 1985; Klein et al. 1986).

From the flux-densities between 50 and $345 \mathrm{GHz}$ we can determine the slope $\alpha$ of the optically thin radio spectrum. Its temporal evolution is shown in Fig. 7. During phase II, it increased from $-2.0 \pm 0.3$ at the onset to $-0.7 \pm 0.1$ at the maximum of $\mathrm{mm} / \mathrm{submm}$ emission. This is consistent with the observation that the emission above $50 \mathrm{GHz}$ was delayed (by $\sim 10-20 \mathrm{~s}$ ) in respect to hard X-rays and microwaves. During phase III, $\alpha$ decreased again to $-2.1 \pm 0.4$ due to the lack of strong emission above $50 \mathrm{GHz}$. As the slope of the optically thin flux-density spectrum is almost independent of the magnetic field $B$ (Dulk 1985), the electron energy spectral index $\delta_{\mathrm{r}}$ can be estimated even though the radio source is inhomogeneous. Using the gyrosynchrotron codes developed by Ramaty (1969), we have calculated the gyrosynchrotron spectra for a cubical homogeneous source. The calculations were carried out for a power-law electron spectrum $\left(N(E)=K E^{\delta_{\mathrm{r}}}\right)$ in the energy range of $0.025-500 \mathrm{MeV}$ and for a viewing angle $\theta$ of 45 degrees, as suggested by the location of the event. For different values of $B$ in the range of $300-1500 \mathrm{G}, \delta_{\mathrm{r}}$ has been estimated for the best fit to the measured spectra above $50 \mathrm{GHz}$. For the two spectra shown in Fig. 6 we obtain $\delta_{\mathrm{r}}=2.3 \pm 0.1$ 


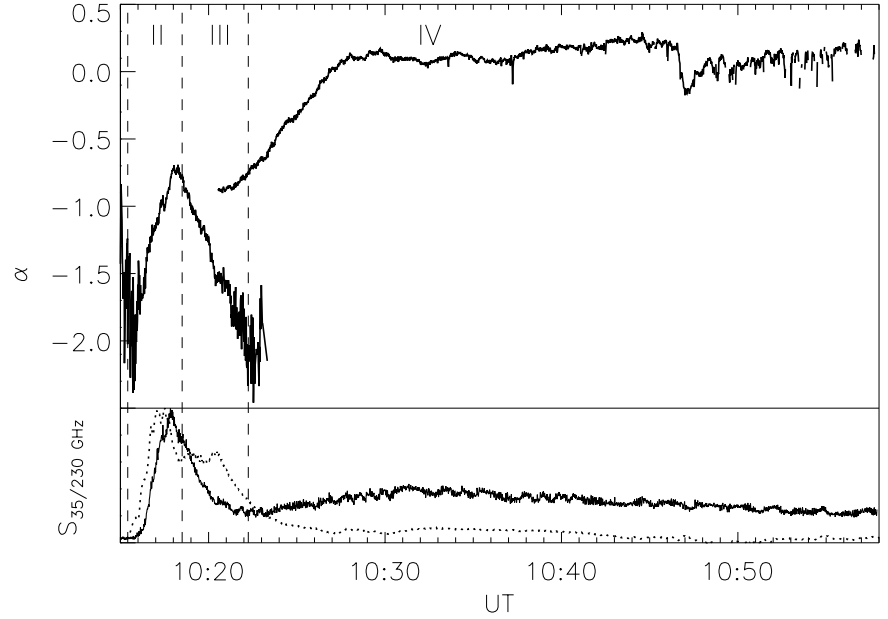

Fig. 7. Temporal evolution of the radio spectral index $\alpha$. During the impulsive phase (II and III), $\alpha$ was determined from the slope of the optically thin spectrum between 50 and $345 \mathrm{GHz}$, after subtraction of the thermal emission component. During the extended phase (IV), all frequencies were taken into account except 35 and $50 \mathrm{GHz}$ that were increasingly affected by bad weather. The sudden break between $\sim 10: 46$ and 10:56 is due to a smaller burst visible only below $35 \mathrm{GHz}$ (cf. Fig. 1). For better orientation the normalised flux densities at 35 and $230 \mathrm{GHz}$ are included (dotted and solid lines, respectively). They also clearly show the delay of the high-frequency emission.

and $3.5 \pm 0.2$ (at 10:17:54 and 10:20:25, respectively). Thus, the energy spectrum of the electrons injected during phase III is significantly softer than during phase II, and/or the high energy cut-off of the radiating electrons is lower during phase III than during phase II (Ramaty et al. 1994).

The electron energy spectrum deduced from radio observations $\left(\delta_{\mathrm{r}}=2.3\right.$ and 3.5$)$ is significantly harder than that derived from the hard X-ray photon spectrum $\left(\delta_{\mathrm{X}}=4.9-5.4\right.$ and 5.1-5.6, see Table 2). This has been observed before in many flares (e.g. Lim et al. 1992; Kundu et al. 1994) and is explained by spectral hardening above a break in the energy spectrum: Most of the hard X-rays with photon energy $\mathrm{E}_{\gamma}$ are emitted by electrons with energies between $\mathrm{E}_{\gamma}$ and $3 \times \mathrm{E}_{\gamma}$ (Kosugi et al. 1988). Thus, the observed hard X-ray emission (below $\sim 200 \mathrm{keV}$ ) stems mainly from lower energy electrons $(\$ 600 \mathrm{keV})$, whereas the millimeter and submillimeter radiation is emitted by electrons at higher energies $(\gtrsim 0.5 \mathrm{MeV})$ (Kundu et al. 1994; Trottet et al. 1998, 2000). For the event under discussion the break energy must be well above several hundred $\mathrm{keV}$, the hard $\mathrm{X}$-ray $/ \gamma$-ray flux of the high energy electrons being too weak to produce any detectable response above $200 \mathrm{keV}$ (Fig. 5). The characteristic energy of electrons radiating synchrotron emission at $345 \mathrm{GHz}$ however is 7-16 MeV for magnetic fields in the 300-1500 G range and a viewing angle of 45 degrees (Dulk 1985).

\section{Radio spectrum during the extended phase}

After the impulsive phase, the flux density at 230 and $345 \mathrm{GHz}$ rose again, reaching a maximum at $\sim 10: 33$ (phase IV). The radio spectrum became rapidly flat $(\alpha \approx 0$ at 10:27, Figs. 7 and 8 ), and afterwards, during the extended phase, slowly increased to 0.2 (10:58). This suggests that the emission is due

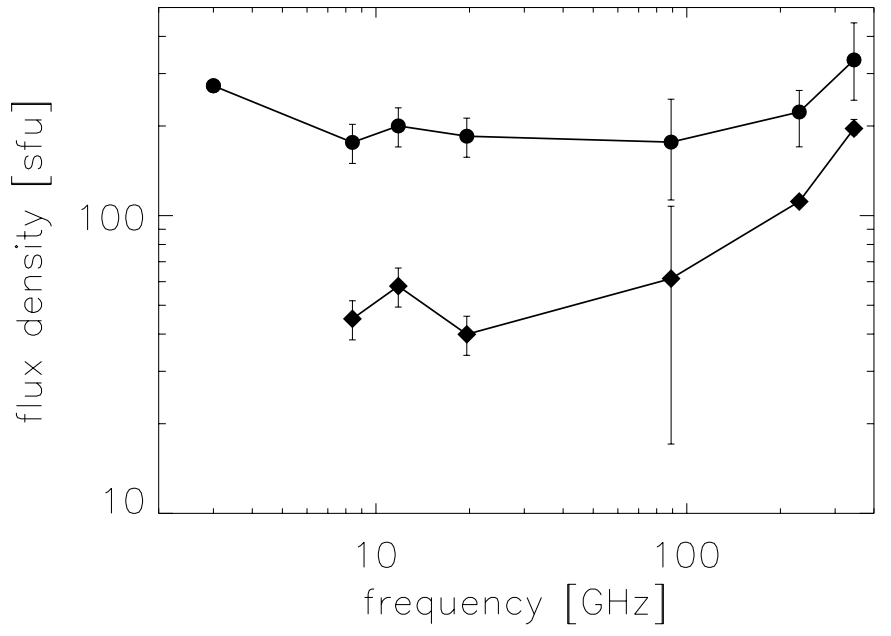

Fig. 8. Nearly flat $(\alpha \approx+0.1)$ spectrum at the time of maximum thermal submm emission (10:33, dots) and the slightly harder $(\alpha \approx+0.3)$ spectrum at the time of the solar maps (11:10, diamonds).

to optically thin thermal bremsstrahlung. Using the temperature $T_{\mathrm{e}}$ and emission measure $E M_{\mathrm{X}}$ derived from GOES measurements, the expected radio flux density at the frequency $v$ becomes

$S_{v}=1.32 \times 10^{-46}\left[24.5+\ln \left(T_{\mathrm{e}}\right)-\ln (v)\right] T_{\mathrm{e}}^{-1 / 2} E M_{\mathrm{X}}$,

where $S_{v}$ is expressed in sfu, $T_{\mathrm{e}}$ in $\mathrm{K}$ and $v$ in $\mathrm{Hz}$ (Pohjolainen et al. 1996a).

The qualitative comparison of the predicted flux densities $S_{v}$ with the observed values shows good agreement (Fig. 9). Both reach their maximum at $\sim 10: 33$. The magnitudes of the measured flux densities, however, are higher than the predicted ones: At frequencies up to $89.4 \mathrm{GHz}$ the observed flux densities are higher by a factor of $\sim 1.5$, at 230 and $345 \mathrm{GHz}$ by a factor of $\sim 2.4$ and $\sim 3.4$ respectively (Fig. 8). At $345 \mathrm{GHz}$ this discrepancy becomes even more pronounced later in the event. Pohjolainen et al. (1996b) analysed a large sample of gradual type bursts and found comparable excesses of the observed $37 \mathrm{GHz}$ emission in $\sim 20 \%$ of the cases. Even larger differences between expected and observed thermal flux densities were reported by Shimabukuro (1970) at $90 \mathrm{GHz}(0.13$ vs. $36 \mathrm{sfu}$ ) and Trottet et al. (2002) at $212 \mathrm{GHz}$ (20 vs. $190 \mathrm{sfu}$ ).

The estimate of the radio flux density from GOESobservations assumes a single-temperature plasma. However, this is almost certainly incorrect for the flare plasma in the solar corona. The soft X-ray emission increases with rising plasma temperature $T_{\mathrm{e}}$ whereas the radio emission decreases (Eq. (3)). Thus, radio observations are more sensitive to cool material than soft X-ray observations. Any significant amount of lower temperature plasma could easily produce a stronger emission in the $\mathrm{mm} / \mathrm{submm}$-range than predicted by the GOESobservations (White \& Kundu 1992). It appears that both the $\mathrm{mm} / \mathrm{submm}$ emission and the soft X-rays are thermal and have a common energy source (resulting in an identical shape of expected and measured time profiles, Fig. 9), but they do not arise from the same thermal electron populations (Shimabukuro 1970; Chertok et al. 1995). The spatial distribution of the lower-temperature plasma may well be different from the one 


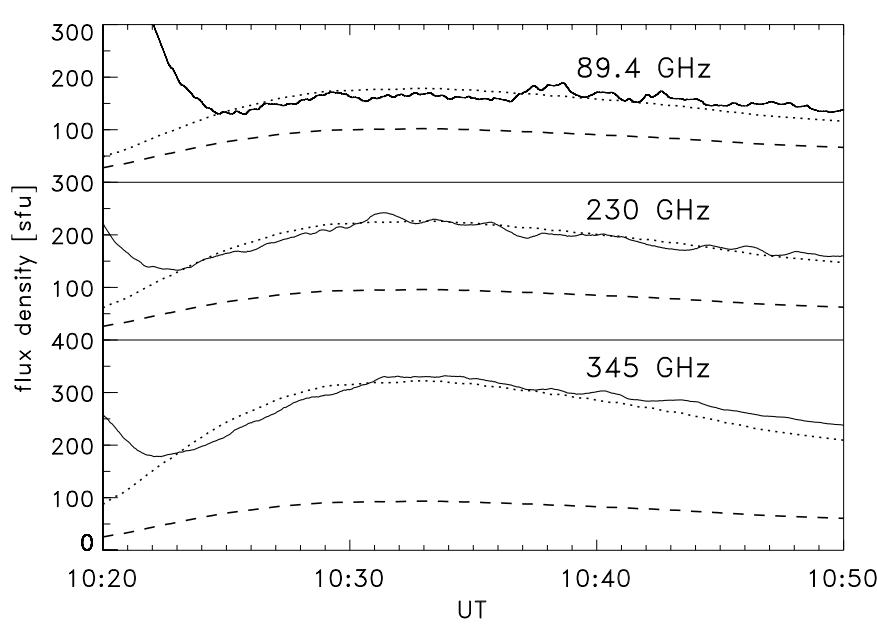

Fig. 9. Extended millimeter and submillimeter thermal emission during phase IV (1 minute average). The solid lines indicate the observed flux density and the dashed ones the flux density predicted from the GOES data. Although the magnitude of the latter is lower the time profiles themselves fit well when scaled by a constant factor (dotted lines).

of the soft X-ray emitting plasma: The last SXT-images before the satellite night (10:39) show a loop of $\sim 15 \times 25 \operatorname{arcsec}^{2}$ (halfpower area), while the solar maps at 11:13 yield source diameters of 42 and 70 arcsec at 230 and $345 \mathrm{GHz}$ respectively. The position of the thermal $\mathrm{mm} / \mathrm{submm}$ source however is in good agreement with the soft X-ray loop. Thus the differences between soft X-rays and $\mathrm{mm} / \mathrm{submm}$ waves can be reconciled if it is assumed that the soft X-rays originate from a hot central core, whereas the $\mathrm{mm} / \mathrm{submm}$ emission arises from cooler plasma in a larger region surrounding the soft X-ray source (Shimabukuro 1972).

The solar maps at 11:13 allow the deconvolution of the size of the thermal source. Assuming Gaussian brightness temperature profiles, the deconvolved source sizes are $42 \pm 20$ and $70 \pm 6$ arcsec at 230 and $345 \mathrm{GHz}$ respectively (Table 1), generally in agreement with previous observations: Shimabukuro (1972) reported source diameters of up to 2.8 arcmin for gradual bursts at $90 \mathrm{GHz}$. Remarkably, the source at $230 \mathrm{GHz}$ is smaller than at $345 \mathrm{GHz}$. The increase in source size with frequency is also found from subreflector scans during the impulsive phase ( $\leq 30$ and $70 \pm 15$ arcsec at 230 and $345 \mathrm{GHz}$ respectively). So far there is no satisfactory explanation: Source asymmetries and inhomogeneities cannot account for the increase of the source size with frequency. Speculatively assuming a low-lying source partially occulted by cool and dense material, we can reasonably account for the observed source sizes and slightly improve the agreement between the observed and modelled spectra. However, there is no confirmation by complementary observations (e.g. $\mathrm{H} \alpha$ filaments). It thus remains to be seen if the observed increase of the source size with frequency is confirmed by future observations or if it is a peculiar feature of the studied flare.

\section{Conclusions}

In this paper we have reported and analysed observations at millimeter and submillimeter wavelengths of a major (GOES class X2.0) solar flare that occurred on April 12, 2001. The observations at 230 and $345 \mathrm{GHz}$ were obtained with the KOSMA telescope and are complemented by our patrol observations, providing an unprecedented spectral coverage from 8.4 to $345 \mathrm{GHz}$.

The event exhibits an impulsive phase with two groups of peaks at $\sim 10: 17: 54$ and 10:20:25 (phases II and III, Fig. 1) and an extended phase lasting at least until 12:45 (phase IV). Emission during the impulsive phase is gyrosynchrotron radiation by nonthermal electrons with a spectral index $\delta_{\mathrm{r}}$ of $2.3 \pm 0.1$ and $3.5 \pm 0.2$ (phases II and III respectively). This is considerably harder than the spectral index $\delta_{\mathrm{X}}$ deduced from the corresponding hard X-ray observations (4.9-5.4 and 5.1-5.6), and thus indicates a flattening of the electron energy spectrum above a break energy of several hundred keV. This event clearly demonstrates the value of $\mathrm{mm} / \mathrm{submm}$ observations as a diagnostic tool for high energy electrons $(\sim 10 \mathrm{MeV}$ for synchrotron emission at $345 \mathrm{GHz}$ ), especially as there is no detectable hard $\mathrm{X}$-ray $/ \gamma$-ray signature above $200 \mathrm{keV}$.

The time-extended component during phase IV represents thermal bremsstrahlung: It exhibits a flat radio spectrum and smooth time profiles, their shape closely following the radio emission predicted from the recorded soft X-rays. The observed absolute flux densities however exceed the computed ones by a factor of $\sim 1.5-3.4$. It appears that both the observed radio emission and the soft $\mathrm{X}$-rays have a common energy source, but are produced at locations with different plasma parameters.

Solar maps and subreflector scans allow us to locate source positions and to estimate for the first time source sizes at 230 and $345 \mathrm{GHz}$ during the thermal phase of a flare. Source positions coincide within 0.2 arcmin and are centred on the hard and soft X-ray sources. However, the observed source diameters reveal an unexpected result as an increase from 230 to $345 \mathrm{GHz}$ was found. The solar maps during the thermal phase yield deconvolved source diameters of $42 \pm 20$ and $70 \pm$ 6 arcsec at 230 and $345 \mathrm{GHz}$ respectively. This effect is also seen in the subreflector scans during the impulsive phase, when the $230 \mathrm{GHz}$ source has a diameter of $\leq 30 \mathrm{arcsec}$, whereas the $345 \mathrm{GHz}$ source diameter is $70 \pm 15$ arcsec. No satisfactory explanation for the increase of the source size with frequency has been found so far. Millimeter/submillimeter observations provide a unique diagnostic tool to examine the energy transport in the corona and lower solar atmosphere during the extended phase of flares. A comprehensive and quantitative analysis including other data such as EUV and optical observations is beyond the scope of this paper, but could shed light on the mechanisms involved.

Acknowledgements. This research is funded by the Swiss National Science Foundation under the grant 2000/063897.00. The KOSMA $3 \mathrm{~m}$ radiotelescope at Gornergrat-Süd Observatory is operated by the University of Cologne and supported by special funding from the Land Nordrhein-Westfahlen. The Observatory is administered by the 
Internationale Stiftung Hochalpine Forschungsstationen Jungfraujoch und Gornergrat, Bern. This research has made also use of Yohkoh data obtained through the Solar Data Analysis Center at the NASA Goddard Space Flight Center. GOES-data was contributed by the U.S. Department of Commerce, NOAA, Space Environment Center, $\mathrm{H} \alpha$ images by Kanzelhöhe observatory (Austria) and $3 \mathrm{GHz}$ radio data by Ondrejov observatory (Czech Republic). We thank the referee Dr. G. Trottet for his comments and useful suggestions that helped to improve the paper.

\section{References}

Akabane, K., Nakajima, H., Ohki, K., Moriyama, F., \& Miyaji, T. 1973, Sol. Phys., 33, 431

Brown, J. C. 1971, Sol. Phys., 18, 489

Chertok, I. M., Fomichev, V. V., Gorgutsa, R. V., et al. 1995, Sol. Phys., 160, 181

Croom, D. L. 1970, Sol. Phys., 15, 414

Croom, D. L. 1979, Jan. 1, Solar Flare Millimetre Data, Appleton Laboratory, Ditton Park, Slough, England

Croom, D. L., \& Powell, R. J. 1969, Nature, 221, 945

Dulk, G. A. 1985, ARA\&A, 23, 169

Kämpfer, N., Magun, A., Feist, D., et al. 1997, in 6th International Symposium on Recent Advances in Microwave Technology Proceedings, Beijing, China

Kaufmann, P., Correia, E., Costa, J. E. R., Zodi Vaz, A. M., \& Dennis, B. R. 1985, Nature, 313, 380

Kaufmann, P., Magun, A., Levato, H., et al. 1999, XXVIth URSI Gen. Ass. Conference, Toronto, Canada

Kawabata, K., Ogawa, H., Takakura, T., et al. 1982, in HINOTORI Symp. on Solar Flares, Inst. Space and Aeronaut. Sci., Tokyo, 168

Klein, K.-L., \& Trottet, G. 1984, A\&A, 141, 67

Klein, K.-L., Trottet, G., \& Magun, A. 1986, Sol. Phys., 104, 243

Kosugi, T., Dennis, B. R., \& Kai, K. 1988, ApJ, 324, 1118

Kramer, C., Degiacomi, C. G., Graf, U. U., et al. 1998, in Proc. SPIEE, 3357,711
Kundu, M. R., White, S. M., Gopalswamy, N., Bieging, J. H., \& Hurford, G. J. 1990, ApJ, 358, L69

Kundu, M. R., White, S. M., Gopalswamy, N., \& Lim, J. 1994, ApJS, 90, 599

Lim, J., White, S. M., Kundu, M. R., \& Gary, D. E. 1992, Sol. Phys., 140,343

Lüthi, T. 1999, Master's thesis, Institute of Applied Physics, University of Bern

Marsh, K. A., Hurford, G. J., Zirin, H., et al. 1981, ApJ, 251, 797

Nakajima, H., Sekiguchi, H., Sawa, M., Kai, K., \& Kawashima, S. 1985, PASJ, 37, 163

Nitta, N., White, S. M., Schmahl, E. J., \& Kundu, M. R. 1991, Sol. Phys., 132, 125

Pohjolainen, S., Valtaoja, E., \& Urpo, S. 1996a, A\&A, 306, 973

Pohjolainen, S., Valtaoja, E., \& Urpo, S. 1996b, A\&A, 314, 947

Ramaty, R. 1969, ApJ, 158, 753

Ramaty, R., Schwartz, R. A., Enome, S., \& Nakajima, H. 1994, ApJ, 436, 941

Raulin, J.-P., White, S. M., Kundu, M. R., Silva, A. V. R., \& Shibasaki, K. 1999, ApJ, 522, 547

Rieger, E., Gan, W. Q., \& Marschhäuser, H. 1998, Sol. Phys., 183, 123

Shimabukuro, F. I. 1970, Sol. Phys., 15, 424

Shimabukuro, F. I. 1972, Sol. Phys., 23, 169

Silva, A. V. R., White, S. M., Lin, R. P., et al. 1996, ApJ, 458, L49

Space Environment Center, 3-day Space Weather Predictions (US Dept. of Commerce, NOAA), available via http://sec.noaa.gov/ftpdir/latest/daypre.txt

Thomas, R. J., Starr, R., \& Crannell, C. J. 1985, Sol. Phys., 95, 323

Trottet, G., Raulin, J.-P., Kaufmann, P., et al. 2002, A\&A, 381, 694

Trottet, G., Rolli, E., Magun, A., et al. 2000, A\&A, 356, 1067

Trottet, G., Vilmer, N., Barat, C., et al. 1998, A\&A, 334, 1099

Vestrand, W. T. 1988, Sol. Phys., 118, 95

Vilmer, N., Trottet, G., Barat, C., et al. 1999, A\&A, 342, 575

White, S. M., \& Kundu, M. R. 1992, Sol. Phys., 141, 347

White, S. M., Kundu, M. R., \& Gopalswamy, N. 1991, ApJ, 366, L43 